\title{
Band Structure Engineering in 2D Photonic Crystal Waveguide with Rhombic Cross-Section Elements
}

\author{
Abdolrasoul Gharaati ${ }^{1}$ and Sayed Hasan Zahraei ${ }^{2}$ \\ ${ }^{1}$ Department of Physics, Payame Noor University, P.O. Box 19395-3697, Tehran, Iran \\ ${ }^{2}$ Nanotechnology Research Institute of Salman Farsi University, P.O. Box 73196-73544, Kazerun, Iran \\ Correspondence should be addressed to Abdolrasoul Gharaati; agharaati@pnu.ac.ir
}

Received 6 February 2014; Revised 14 April 2014; Accepted 15 April 2014; Published 12 May 2014

Academic Editor: José Luís Santos

Copyright (C) 2014 A. Gharaati and S. H. Zahraei. This is an open access article distributed under the Creative Commons Attribution License, which permits unrestricted use, distribution, and reproduction in any medium, provided the original work is properly cited.

\begin{abstract}
Two-dimensional photonic crystal (2D PhC) waveguides with square lattice composed of dielectric rhombic cross-section elements in air background, by using plane wave expansion (PWE) method, are investigated. In order to study the change of photonic band gap (PBG) by changing of elongation of elements, the band structure of the used structure is plotted. We observe that the size of the PBG changes by variation of elongation of elements, but there is no any change in the magnitude of defect modes. However, the used structure does not have any TE defect modes but it has TM defect mode for any angle of elongation. So, the used structure can be used as optical polarizer.
\end{abstract}

\section{Introduction}

PhCs are class of media represented by natural or artificial structures with periodic modulation of the refractive index [1-3]. Such optical media have some peculiar properties which gives an opportunity for a number of applications to be implemented on their basis. In $2 \mathrm{D} \mathrm{PhCs}$, the periodic modulation of the refractive index occurs in two directions, while in one other direction structure is uniform. When the refractive index contrast between elements of the PhC and background is high enough, a range of frequencies exists for which propagation is forbidden in the $\mathrm{PhC}$ and called photonic band gap (PBG). The PBG depends upon the arrangement and shape of elements of the $\mathrm{PhC}$, fill factor, and dielectric contrast of the two mediums used in forming $\mathrm{PhC}$. The most important feature of PhCs is ability to support spatially electromagnetic localized modes when a perfectly periodic $\mathrm{PhC}$ has spatial defects [4-6]. In recent years, a lot of researches are devoted to study $2 \mathrm{D} \mathrm{PhC}$ with circular, square, and elliptic cross-section elements [7, 8]. However, less work was devoted to study of $\mathrm{PhC}$ with rhombic cross-section elements. In this paper, we study band structure for 2D PhC waveguide with dielectric rhombic cross-section elements with a square lattice and how band structure is affected by elongating of elements.

\section{PWE Method and Numerical Analysis}

We consider 2D PhC waveguide as shown in Figure 1(a), consisting of a square lattice of GaAs rhombic cross-section elements in air background, having a lattice constant of $a=$ $815 \mathrm{~nm}$. The rhombuses have $0.4 a$ side and a refractive index of $n_{a}=3.37$ [8]. The waveguide core is formed by substitution of a row of rhombuses with a row of different rhombuses with refractive index $n_{d}=1$ and $0.4 a$ side along the $y$ direction. Figure 1(b) shows the unit cell for the structure used which is composed of the elements as shown in Figure 1(c) [1].

To obtain the band structure of the considered $2 \mathrm{D} \mathrm{PhC}$ waveguide, the PWE method has been employed $[1,5]$. Based on the symmetry considerations, the general form of the magnetic field vector of a TE-polarized mode and the electric field vector of a TM-polarized mode expanded into plane wave vector $\vec{K}$ with respect to the $2 \mathrm{D}$ reciprocal lattice vector $\vec{G}$, labeled with a Bloch wave number $k_{y}$, which is given by [1]. 


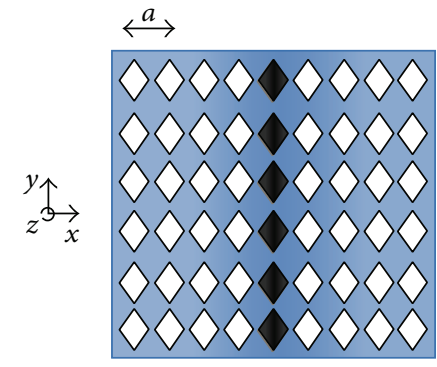

(a)

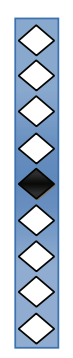

(b)

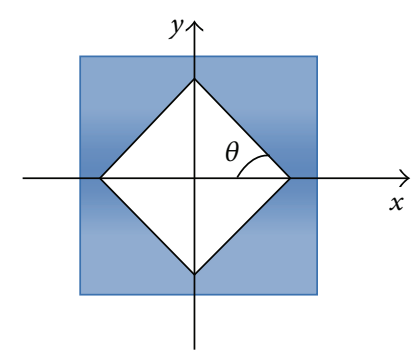

(c)

Figure 1: (a) 2D PhC waveguide, (b) the unit cell of the 2D PhC waveguide, and (c) the element of the unit cell.

For TE-polarized mode,

$$
\begin{aligned}
\vec{H} & =\left(0,0, H_{z, k_{y}}(x, y)\right), \\
H_{z, k_{y}}(\vec{r}) & =\sum_{\vec{G}} \int_{-}^{\frac{\pi}{a}} \frac{\pi}{a} d k_{x} H_{z}(\vec{K}+\vec{G}) \exp (i(\vec{K}+\vec{G}) \cdot \vec{r}) .
\end{aligned}
$$

For TM-polarized mode,

$$
\begin{aligned}
\vec{E} & =\left(0,0, E_{z, k_{y}}(x, y)\right), \\
E_{z, k_{y}}(\vec{r}) & =\sum_{\vec{G}} \int_{-\pi / a}^{\pi / a} d k_{x} E_{z}(\vec{K}+\vec{G}) \exp (i(\vec{K}+\vec{G}) \cdot \vec{r}),
\end{aligned}
$$

where $\vec{H}, \vec{E}, \vec{G}$, and $\vec{K}$ are magnetic field vector, electric field vector, $2 \mathrm{D}$ reciprocal lattice vector, and plane wave vector, respectively. The sum and integral are taken over the first Brillouin zone of the $2 \mathrm{D} \mathrm{PhC}$ waveguide used $[1,5]$.

Solving Maxwell's equations in CGS unit for the magnetic and electric fields leads to the following vector wave equations:

$$
\begin{aligned}
\omega^{2} \vec{H} & =\vec{\nabla} \times\left(\frac{1}{\varepsilon(\vec{r})} \vec{\nabla} \times \vec{H}\right), \\
\omega^{2} \vec{E} & =\frac{1}{\varepsilon(\vec{r})} \vec{\nabla} \times(\vec{\nabla} \times \vec{E}),
\end{aligned}
$$

where $\varepsilon(\vec{r})$ is the dielectric function of the unit cell.

Substituting (1) in the vector wave (3) and (2) in the vector wave (4), we get two eigenvalue problems for the square of frequency $\omega$ for each polarized mode

For TE-polarized mode,

$$
\begin{aligned}
\omega^{2}\left(k_{y}\right) & H_{z}(\vec{K}+\vec{G}) \\
= & -\sum_{\vec{G}} \int_{-\pi / a}^{\pi / a} d k_{x}^{\prime} \kappa\left(\vec{K}+\vec{G}-\vec{K}^{\prime}-\vec{G}^{\prime}\right) \\
& \times\left[\left(\vec{K}^{\prime}+\vec{G}^{\prime}\right) \cdot\left(\vec{K}+\vec{G}-\vec{K}^{\prime}-\vec{G}^{\prime}\right)\right] H_{z}\left(\vec{K}^{\prime}+\vec{G}^{\prime}\right) .
\end{aligned}
$$

For TM-polarized mode,

$$
\begin{aligned}
\omega^{2}\left(k_{y}\right) & E_{z}(\vec{K}+\vec{G}) \\
= & -\sum_{\vec{G}} \int_{-\pi / a}^{\pi / a} d k_{x}^{\prime} \kappa\left(\vec{K}+\vec{G}-\vec{K}^{\prime}-\vec{G}^{\prime}\right) \\
& \times\left|\left(\vec{K}^{\prime}+\vec{G}^{\prime}\right)\right|^{2} E_{z}\left(\vec{K}^{\prime}+\vec{G}^{\prime}\right) .
\end{aligned}
$$

That $\kappa(\vec{k}+\vec{G})$ is the Fourier expansion of the inverse dielectric function of $2 \mathrm{D} \mathrm{PhC}$ waveguide that is written as

$$
\kappa(\vec{K}+\vec{G})=\frac{1}{S_{\text {unit cell }}} \int_{\text {unit cell }} d \vec{r} \frac{1}{\varepsilon(\vec{r})} \exp (-i(\vec{K}+\vec{G}) \cdot \vec{r}) .
$$

That integral is taken over the unit cell in Figure 1(b). For a given value of a Bloch wave number $k_{y}$ as propagation constant $\beta$, (5) and (6) constitute two eigenvalue problems with respect to the square of frequency $\omega\left(k_{y}\right)$. Finally, using a trapezoidal approximation of the $1 \mathrm{D}$ integral $k_{x}^{\prime}$ and the numerical solutions for (5) and (6), we get the band structure of the structure used [1]. The computation method used for implementation of PWE method for $2 \mathrm{D} \mathrm{PhC}$ waveguide is similar to the one which is used for the computation of the band structure of strictly periodic PhC. There is some essential difference in the structure parameters definitions $[1,2]$. First in 2D PhC waveguide the unit cell consists of several $\mathrm{PhC}$ elements rather than one. The defect of periodic structure is also introduced to form the waveguide core. Also, in case of 2D PhC band structure computation, we set the $k$ path to pass through all high symmetry points of the Brillouin zone. However, as we have considered in this section, computation of the $2 \mathrm{D} \mathrm{PhC}$ waveguide band structure requires transversal wave vector consideration only. The longitudinal component stays in this case for the propagation constant and the propagation constant is limited by the boundaries of the Brillouin zone. One more difference from strictly periodic $\mathrm{PhC}$ is the definition of the reciprocal lattice vectors set [1$3]$.

\section{Elongation of the Rhombuses}

According to Figure 2, we can change the elongation angle $\theta$ that it makes with $x$ axis, for transformation of rhombuses. 


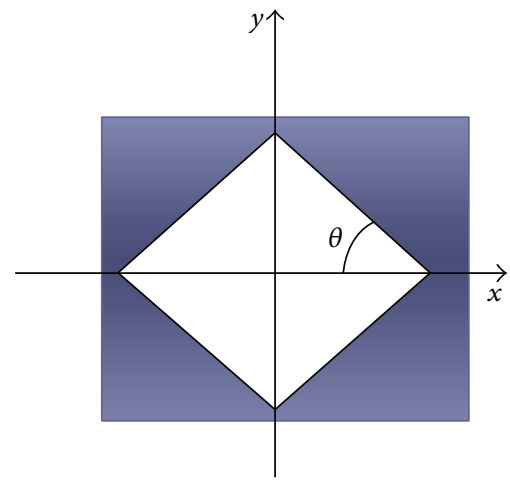

FIGURE 2: Schematic elongation angle $\theta$ that it makes with $x$ axis in the unit cell.

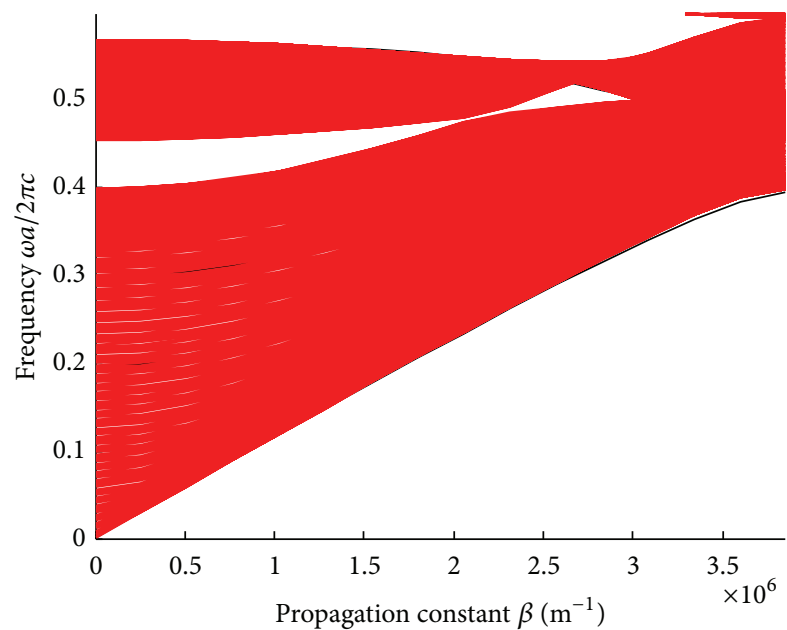

(a)

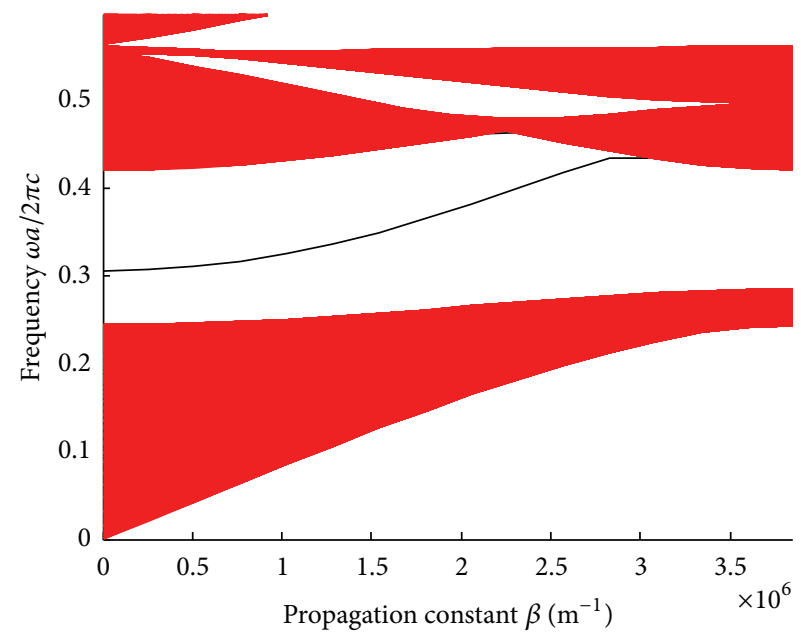

(b)

FIgURE 3: Band structures for elongation angle $\theta=\pi / 4 \mathrm{rad}$ for (a) TE-polarized mode and (b) TM-polarized mode.

By changing of the elongation angle $\theta$, when the definition of the unit cell is being made in discrete form by setting values of inversed dielectric function to mesh nodes, we define the borders of rhombuses in each element of the unit cell as function of the elongation angle $\theta$ as

$$
\begin{aligned}
& |y+\tan \theta x|=0.4 a(\sin \theta), \\
& |y-\tan \theta x|=0.4 a(\sin \theta) .
\end{aligned}
$$

And we change the elongation angle $\theta$ and get the band structure for any angle $\theta$.

\section{Band Structures}

First, we plot the band structure for the 2D PhC waveguide composed of square lattice of GaAs rhombic cross-section elements with side $d=326 \mathrm{~nm}$ and refractive index $n_{a}=3.35$ in air background with a row of line defects, for both TE- and TM-polarized modes. The results are shown in Figure 3 for the elongation angle $\theta=\pi / 4 \mathrm{rad}$. The filled areas in Figure 3 are the continuum of states of the perfectly periodic $2 \mathrm{D} \mathrm{PhC}$ which the $2 \mathrm{D} \mathrm{PhC}$ waveguide is made from. All radiations with frequencies which hit these areas (with red color) will be able to propagate inside the $\mathrm{PhC}$ surrounding the waveguide core. But the radiations with frequencies which lie in the PBG (with white color) do not leak into the surrounding periodic media, so that radiations are guided through the waveguide core and are called defect modes [1-4].

In order to study how band structure is affected by elongating of elements, we change the angle $\theta$ and plot the band structure for a few important angles of elongations. Figure 4 shows the band structures for the elongation angles $\theta(\pi / 6 \& \pi / 3 \mathrm{rad})$ for both TE- and TM-polarized modes. From numerical results in Figures 3 and 4, it is evident that, by increasing the elongation angle, magnitude of defect modes will be constant, but the PBG width increases. Although, for the case TE, there is no defect mode, the structure can be used as optical polarizer waveguide (OPW), which has TM defect mode and does not have TE defect mode. So, the structure transmits one state of polarization and blocks TE defect mode [7-13]. Calculations were performed for two important angles of elongation and all our computational results for any angle confirm these results. 


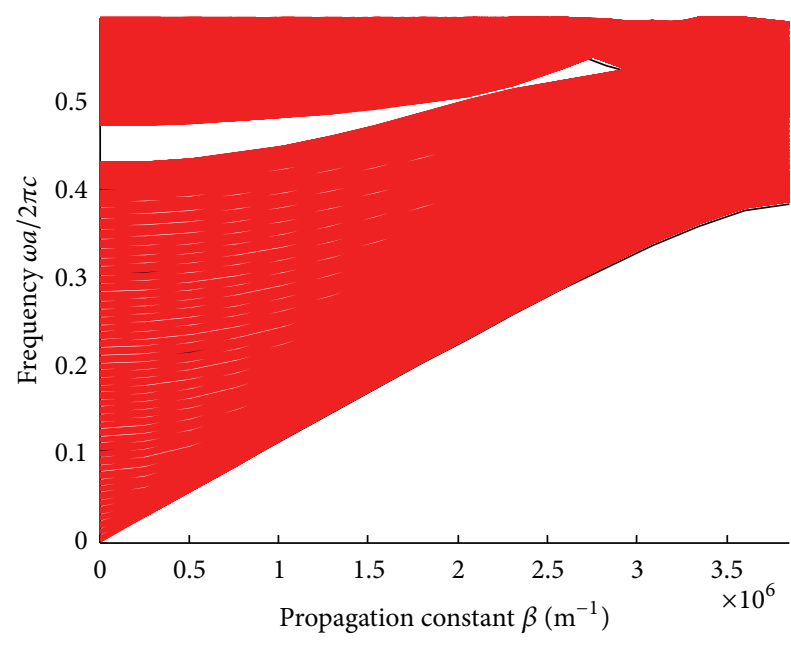

(a)

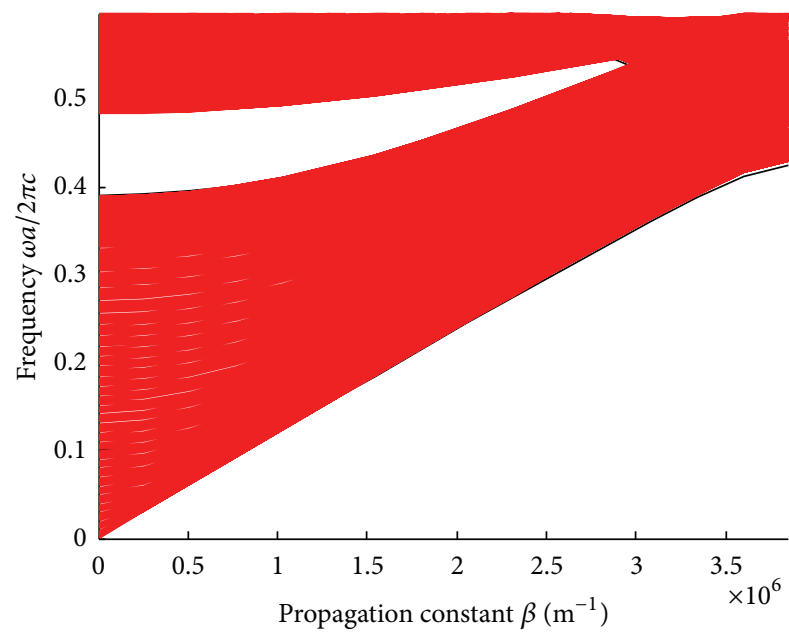

(c)

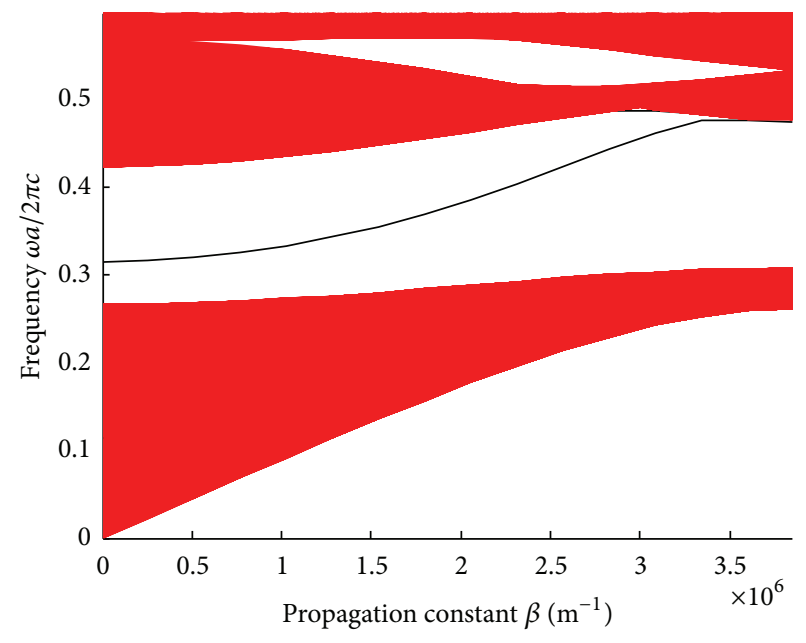

(b)

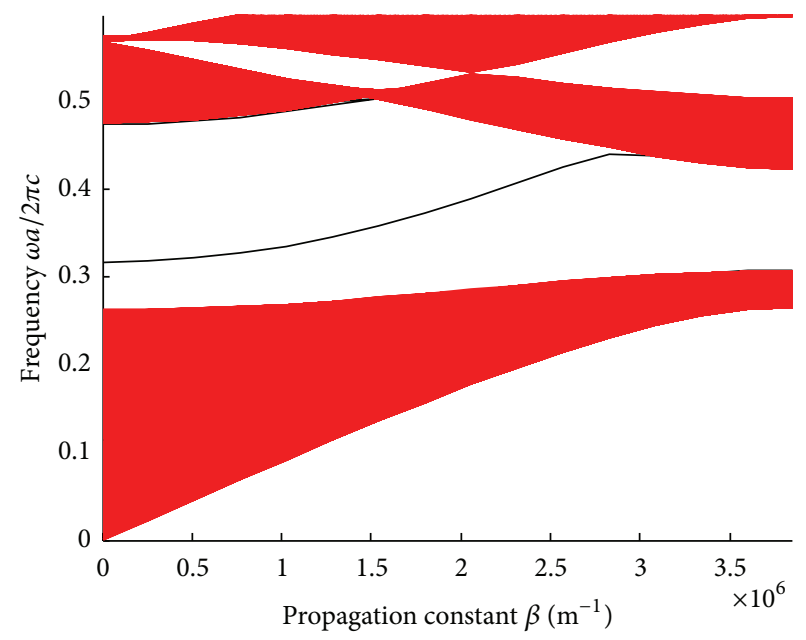

(d)

FIgURE 4: Band structure for $\theta=\pi / 6$ rad in (a) TE mode and (b) TM mode and for $\theta=\pi / 3$ rad in (c) TE mode and (d) TM mode.

\section{Conclusion}

Using PWE method, we have studied band structure for 2D $\mathrm{PhC}$ waveguide with dielectric rhombic cross-section elements in air background. Less works were devoted to study of $\mathrm{PhC}$ with rhombic cross-section elements. So, we considered variations of the elements elongation for the used structure. Numerical results show that, by increasing in the elongation of elements, magnitude of the defect modes remains constant but the size of PBG increases. Also, the used 2D PhC waveguide blocks TE defect mode and transmits TM modes. So, this kind of structure can be used as optical polarizer waveguide.

\section{Conflict of Interests}

The authors declare that there is no conflict of interests regarding the publication of this paper.

\section{Acknowledgment}

This work has been financially supported by Payame Noor University (PNU) I. R. of Iran.

\section{References}

[1] M. Skorobogatiy and J. Yang, Fundamentals of Photonic Crystal Guiding, Cambridge University Press, 2009.

[2] K. Sakoda, Optical Properties of Photonic Crystals, Springer, Berlin, Germany, 2001.

[3] J. D. Joannopoulos, S. G. Johnson, J. N. Winn, and R. D. Meade, Photonic Crystals Molding the Flow of Light, Princeton University Press, 2008.

[4] B. E. A. Saleh and M. C. Teich, Fundamentals of Photonic, WileyInterscience, New York, NY, USA, 2007.

[5] Y. Kalra and R. K. Sinha, "Photonic band gap engineering in 2D photonic crystals," Pramana-Journal of Physics, vol. 67, no. 6, pp. 1155-1164, 2006. 
[6] S. Robinson and R. Nakkeeran, "PCRR based band pass filter for C and L+U bands of ITU-T G.694.2 CWDM systems," Optical and Photonic Journal, vol. 1, no. 3, pp. 142-149, 2011.

[7] R. Stopper, H. J. W. M. Hoekstra, R. M. De Ridder, E. Van Groesen, and F. P. H. Van Beckum, "Numerical studies of 2D photonic crystals: waveguides, coupling between waveguides and filters," Optical and Quantum Electronics, vol. 32, no. 6, pp. 947-961, 2000.

[8] A. V. Dyogtyev, I. A. Sukhoivanov, and R. M. De La Rue, "Photonic band-gap maps for different two dimensionally periodic photonic crystal structures," Journal of Applied Physics, vol. 107, no. 1, Article ID 013108, 7 pages, 2010.

[9] R. K. Sinha and Y. Kalra, "Design of optical waveguide polarizer using photonic band gap," Optics Express, vol. 14, no. 22, pp. 10790-10794, 2006.

[10] I. Guryev, I. A. Sukhoivanov, S. Alejandro-Izquierdo et al., "Analysis of integrated optics elements based on photonic crystals," Revista Mexicana de Fisica, vol. 52, no. 5, pp. 453-458, 2006.

[11] T. Liu, A. R. Zakharian, M. Fallahi, J. V. Moloney, and M. Mansuripur, "Design of a compact photonic-crystal-based polarizing beam splitter," IEEE Photonics Technology Letters, vol. 17, no. 7, pp. 1435-1437, 2005.

[12] M. Bayindir, E. Cubukcu, I. Bulu, T. Tut, E. Ozbay, and C. M. Soukoulis, "Photonic band gaps, defect characteristics, and waveguiding in two-dimensional disordered dielectric and metallic photonic crystals," Physical Review B, vol. 64, no. 19, Article ID 195113, 7 pages, 2001.

[13] M. J. A. De Dood, E. Snoeks, A. Moroz, and A. Polman, "Design and optimization of 2D photonic crystal waveguides based on silicon," Optical and Quantum Electronics, vol. 34, no. 1-3, pp. 145-159, 2002. 

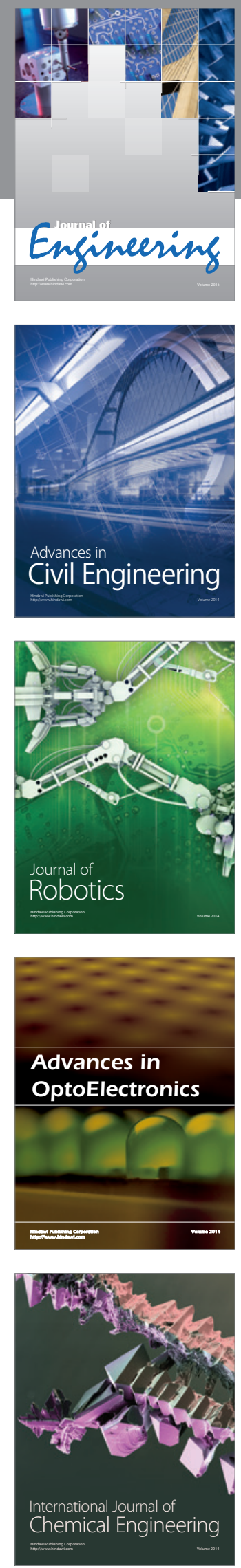

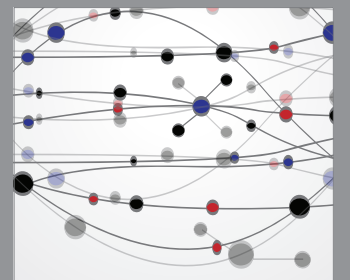

The Scientific World Journal
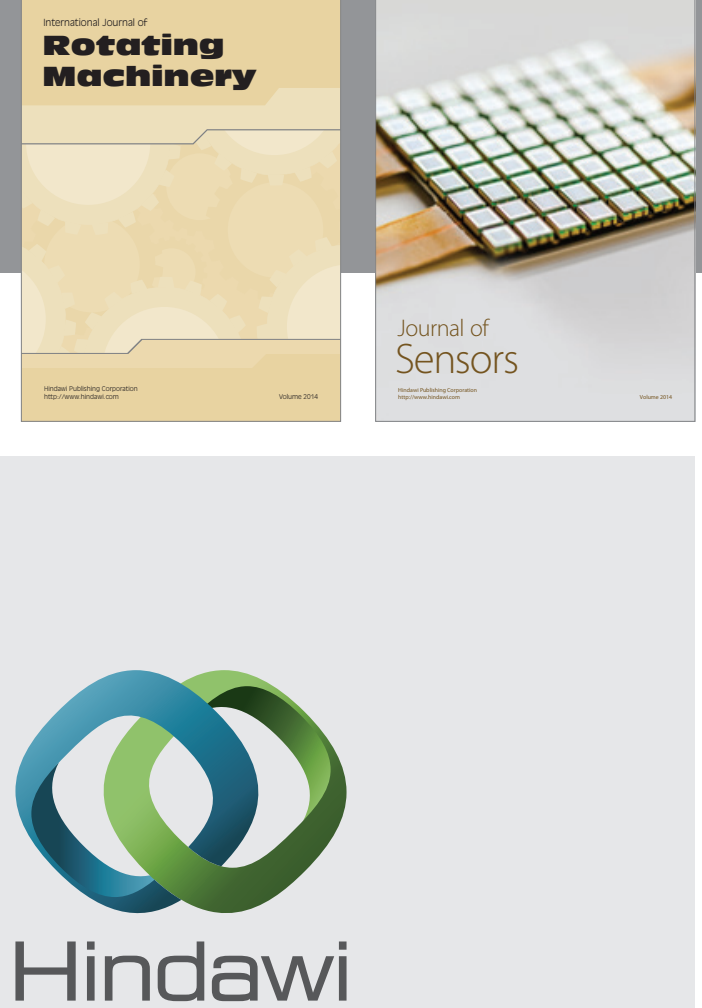

Submit your manuscripts at http://www.hindawi.com
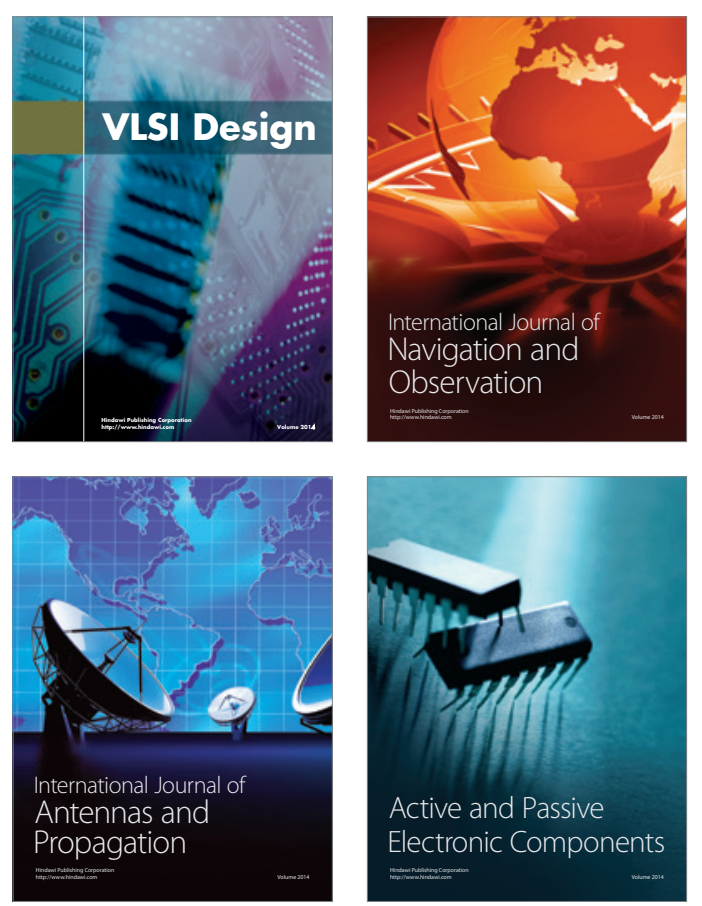
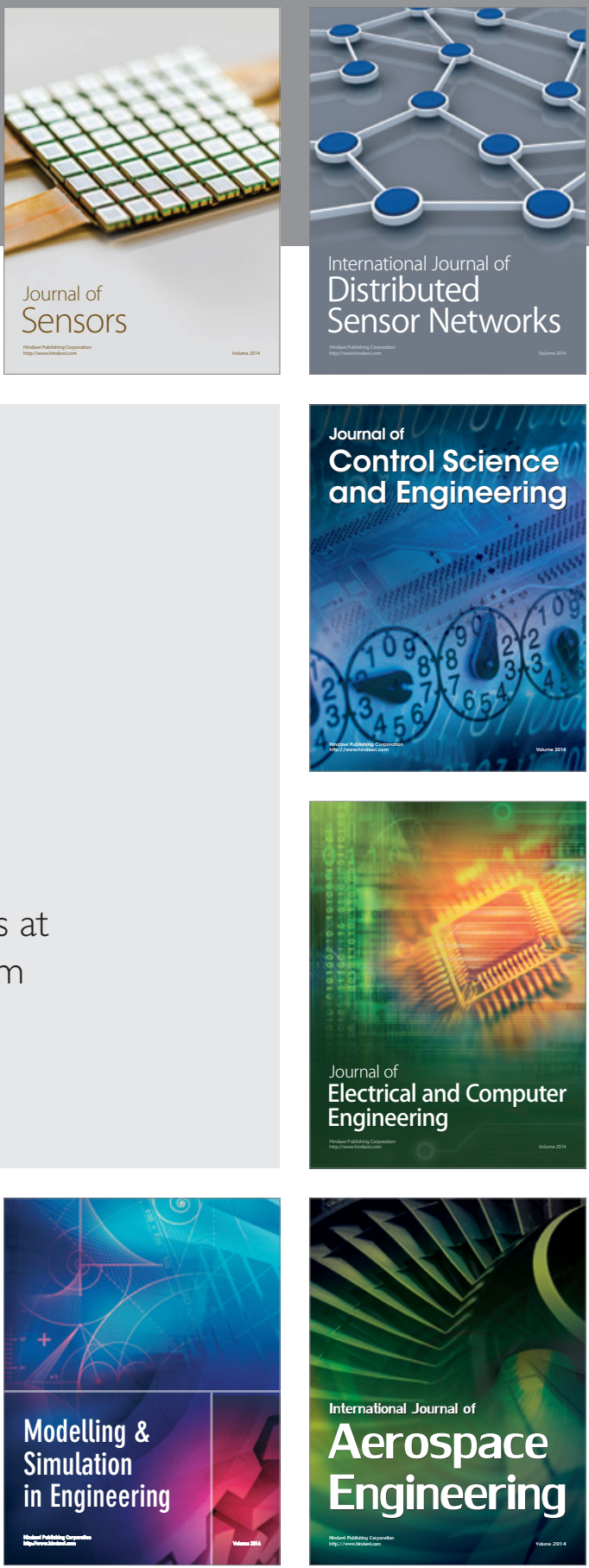

Journal of

Control Science

and Engineering
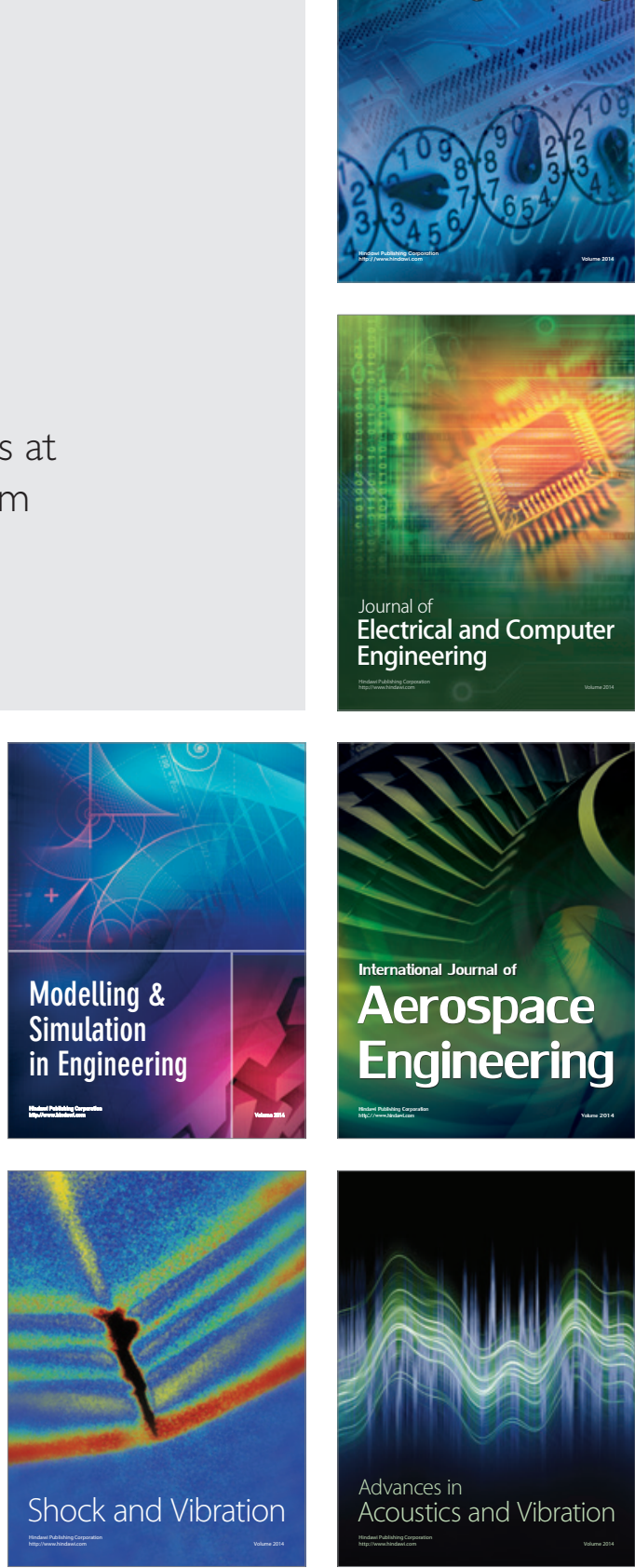